\title{
Spectroscopic Ellipsometry of Porphyrin Adsorbed in Porous Silicon
}

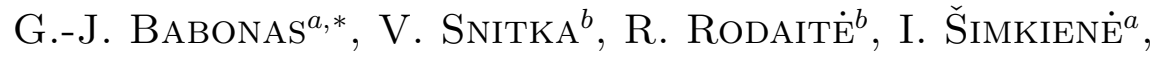 \\ A. RĖZA ${ }^{a}$ AND M. TREIDERIS ${ }^{a}$ \\ ${ }^{a}$ Semiconductor Physics Institute, Goštauto 11, 01108 Vilnius, Lithuania \\ ${ }^{b}$ Kaunas University of Technology, Studentu̧ 65, 51369 Kaunas, Lithuania \\ Aqueous solution of meso-tetra(4-sulfonatophenyl)porphine was de- \\ posited on electrochemically etched $n$-Si wafers. The morphology of the hy- \\ brid systems was investigated by scanning electron microscope and atomic \\ force microscope techniques. The optical response of the hybrid systems \\ was studied by spectroscopic ellipsometry in the range of $1-5 \mathrm{eV}$. Particu- \\ lar features in adsorption process were revealed for meso-tetra(4-sulfonato- \\ phenyl)porphine deposited on variously chemically treated Si substrates. It \\ was found that porphyrin J-aggregates can be intercalated into large pores \\ formed in a bulk $n$-Si as well as into nanopores of luminescent oxide layer.
}

PACS numbers: 78.68.+m, 61.43.-j, 61.43.Gt

\section{Introduction}

During last decade porphyrins have been extensively studied due to their importance in biological processes and perspectives in various technical applications such as catalysis, photodynamic therapy, biosensors, etc. For example, tetraphenyl porphyrin based thin film transistors were manufactured [1] on thermally oxidized Si substrates. On the other hand, nanostructured porous silicon (por-Si) is a very promising biomaterial, in particular, for a development of devices that are to be linked to the biological systems [2]. From this point of view, the adsorption processes are to be studied making use of non-destructive methods such as ellipsometry. Spectroscopic ellipsometry was shown [3] to be efficient tool to study protein adsorption in por-Si layers.

In this work the system consisting of porhyrin on por-Si layers was studied by means of structural and optical investigations. The regularities of adsorption of porphyrin water solution on the surface of variously chemically treated Si substrates were examined.

*corresponding author; e-mail: jgb@pfi.lt 


\section{Experimental}

The aqueous solution of porphyrin of concentration $1 \times 10^{-4} \mathrm{M}$ was prepared by dissolving meso-tetra(4-sulfonatophenyl)porphine $\left(\mathrm{TPPS}_{4}\right)$ tetrasodium salt. Adding $0.1 \mathrm{M} \mathrm{HCl}$, the solution of $\mathrm{pH}=1$ was made. In the aqueous medium of this acidity the J-aggregates are formed [4]. The samples were stored at room temperature for 10 days to achieve a stable state with respect to the formation of large J-aggregates.

The (100)-oriented wafers of $n$-Si $(0.5 \Omega \mathrm{cm})$ were used as substrates. The wafers were degreased by dimethyl formamide and washed by methanol. Porous layers were produced by electrochemical etching in electrolyte $\mathrm{HF}: \mathrm{C}_{2} \mathrm{H}_{5} \mathrm{OH}(1: 1)$ at $15-35 \mathrm{~mA} / \mathrm{cm}^{2}$ for $15 \mathrm{~min}$ illuminating the back surface of etched sample with $50 \mathrm{~W}$ halogen lamp $(1200 \mathrm{~lx})$. A droplet $(0.1 \mathrm{~mL})$ of porphyrin water solution was put on the sample surface and dried under normal conditions. The morphology of composite structures was investigated by scanning electron microscope (SEM) and atomic force microscope (AFM).

Spectroscopic ellipsometric measurements were carried out by means of the photometric ellipsometer with rotating analyzer operating in the range of $1-5 \mathrm{eV}$. The measurement results characterize the optical response of the system under investigation in terms of a complex reflection $\rho$ defined as the ratio of complex amplitude reflection coefficients $R_{\mathrm{p}}$ and $R_{\mathrm{s}}$ for light polarized parallel (p) and perpendicular $(\mathrm{s})$ to the plane of incidence:

$$
\rho=\frac{R_{\mathrm{p}}}{R_{\mathrm{s}}}=\tan \Psi \exp (\mathrm{i} \Delta),
$$

where $\Psi$ and $\Delta$ are ellipsometric parameters.

The experimental data were interpreted in the approximation of pseudodielectric function $\langle\varepsilon\rangle$, in which the optical response of a complex system (air-filmsubstrate) is considered as that for isotropic media [5]:

$$
\langle\varepsilon\rangle=\sin ^{2} \Theta\left[\left(\frac{1-\rho}{1+\rho}\right)^{2} \tan ^{2} \Theta+1\right],
$$

where $\Theta$ is the angle of light incidence. As a rule, ellipsometric measurements were carried out at $\Theta=70^{\circ}$.

\section{Results and discussion}

The results of optical studies are illustrated by Figs. 1 and 2. In the photon energy range under consideration the absorbance spectrum of investigated $\mathrm{TPPS}_{4}$ aqueous solution (curve 1 in Fig. 1) consisted of three strongest absorption bands located at $1.75,2.53$, and $2.90 \mathrm{eV}$. The energy and relative intensities of the bands were in good agreement with reference data (see, e.g., [4]). The presence of the first two bands confirmed the formation of J-aggregates [4]. The optical response 


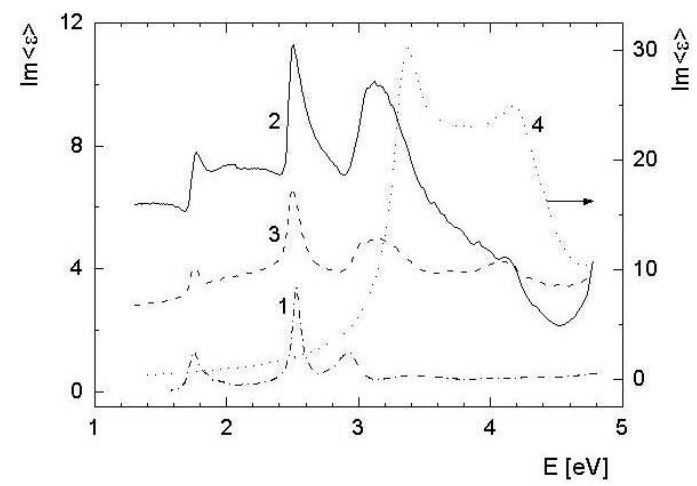

Fig. 1. Optical spectra of $\mathrm{TPPS}_{4}$ aqueous solution (1), hybrid samples of $\mathrm{TPPS}_{4}$ on $c$-Si (2), TPPS 4 on por-Si with removed oxide layer (3) and $c$-Si substrate (4). Absorbance of $\mathrm{TPPS}_{4}$ aqueous solution (1) and imaginary part of pseudodielectric function (2-4) are shown.

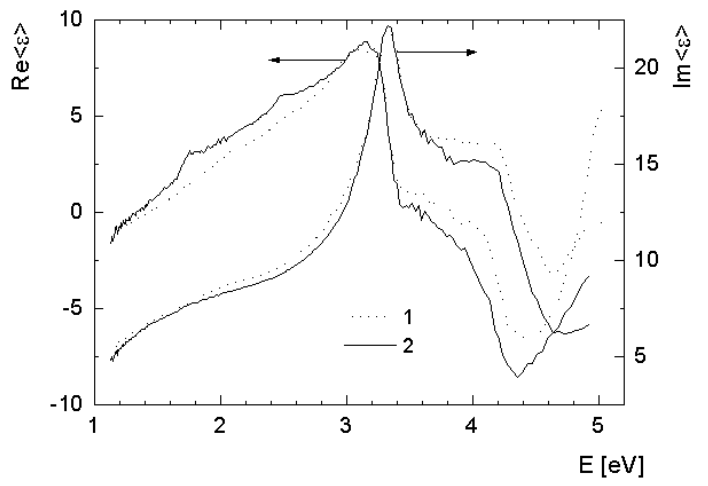

Fig. 2. Pseudodielectric function spectra of as-grown por-Si (1) and hybrid sample of $\mathrm{TPPS}_{4}$ deposited on por-Si (2).

of a control hybrid sample $\mathrm{TPPS}_{4}$ on glass was dominated by porphyrin. The fine structure of the spectra for hybrid sample $\mathrm{TPPS}_{4} /$ glass was qualitatively the same though the relative intensity of the absorption bands was slightly changed. For a latter hybrid sample the spectroscopic ellipsometry data were analyzed in a three-media model and the dielectric function spectra of $\mathrm{TPPS}_{4}$ were determined for the first time. The imaginary part of dielectric function was equal to $\sim 2.5$ and $\sim 5.5$ at the maximum of the bands at 1.75 and $2.53 \mathrm{eV}$, respectively. The uncertainty in the evaluation of numerical values is mainly due to the variation of $\mathrm{TPPS}_{4}$ layer thickness.

The fine structure of pseudodielectric function spectra $\langle\varepsilon\rangle(E)$ for $\mathrm{TPPS}_{4}$ deposited on crystalline Si wafer (curve 2 in Fig. 1) was mainly caused by porphyrin in the region of lower photon energies. Some interaction between $\mathrm{TPPS}_{4}$ and crystalline $n$-Si (100)-oriented surface can be proposed from the lineshape analysis 
in the range of 2.8-3.5 eV due to overlap of the characteristic peaks in both materials. At higher energies the fine structure of the optical response is caused by $\mathrm{Si}$ (curve 4 in Fig. 1). The contribution of $\mathrm{TPPS}_{4}$ can be modeled as that for a transparent film up to the energy of strong electronic excitations starting at $E>5 \mathrm{eV}$.

Curve 3 in Fig. 1 illustrates the optical response of hybrid sample consisting of $\mathrm{TPPS}_{4}$ deposited on por-Si with oxide layer removed by etching for $5 \mathrm{~min}$ in a $30 \%$ water solution of $\mathrm{KOH}$. Immediately before deposition of $\mathrm{TPPS}_{4}$, the native oxide was removed by etching for $1 \mathrm{~min}$ in $\mathrm{HF}: \mathrm{H}_{2} \mathrm{O}$ (1:1). The lineshape difference for optical features due to porphirin absorption bands in curves 2 and 3 indicates a different interaction of $\mathrm{TPPS}_{4}$ with crystalline and porous Si surface. The regularities of the influence of $\mathrm{TPPS}_{4}$-deposition are well illustrated in the difference spectra between initial and hybrid samples.

The pseudodielectric function spectra for hybrid sample of $\mathrm{TPPS}_{4}$ deposited on as-formed anodically etched por-Si is presented in Fig. 2. As is seen, the optical features due to $\mathrm{TPPS}_{4}$ were considerably masked though clearly resolved in the region of $\sim 1.7$ and $\sim 2.5 \mathrm{eV}$.

AFM studies of $\mathrm{TPPS}_{4} /$ glass samples have shown that parallel fiber-like structures of $0.5-1.0 \mu \mathrm{m}$ in width and $100-200 \mathrm{~nm}$ in height were formed. The structures on the glass surface present a stacking of $\mathrm{TPPS}_{4}$ mesoaggregates consisting of oblong nanoparticles of $\oslash 100-200 \mathrm{~nm}$ and $15-30 \mathrm{~nm}$ in height.

SEM studies have shown that a $\mathrm{TPPS}_{4}$ layer on the surface of crystalline $\mathrm{Si}$ was not uniform. Rectangular-like openings of $1-5 \mu \mathrm{m}$ in size were formed. The openings were oriented along crystallographic axes indicating a strong interaction of $\mathrm{TPPS}_{4}$ with (100)-surface of crystalline $n$-Si. As follows from AFM observations, the fibers similar to those on glass substrate were formed on the surface of crystalline Si (Fig. 3a) though in this case the flocculated structure of fibers was not so clearly developed. The width and height of fibers were approximately equal to 250 and $30-50 \mathrm{~nm}$, respectively. These fibers were significantly larger than those [4] formed on crystalline $\mathrm{Si}$ from aqueous solution with a lower $\left(5 \times 10^{-5} \mathrm{M}\right)$ concentration of $\mathrm{TPPS}_{4}$.

The structure of por-Si was typical [6] for $n$-Si. The morphology of por-Si with removed oxide layer investigated by SEM has shown that the pores of size $\sim 2-4 \mu \mathrm{m}$ and depth $\sim 15 \mu \mathrm{m}$ were formed during electrochemical etching. As follows from Fig. 3b, the fiber-like structure was not formed and porphyrin aggregates were located predominantly inside the pores.

The structural and optical studies have shown that adsorption process of porphyrin aqueous solution was different for various Si surfaces. Large mesoaggregates of $\mathrm{TPPS}_{4}$ were formed on the surface of glass and crystalline Si resulting in the change of the characteristic absorption bands. Analyzing the whole set of data it was concluded that porphyrin J-aggregates were intercalated into large pores of size $2-4 \mu \mathrm{m}$ in $n$-Si bulk as well as in the luminescent layer, in which nanopores of 

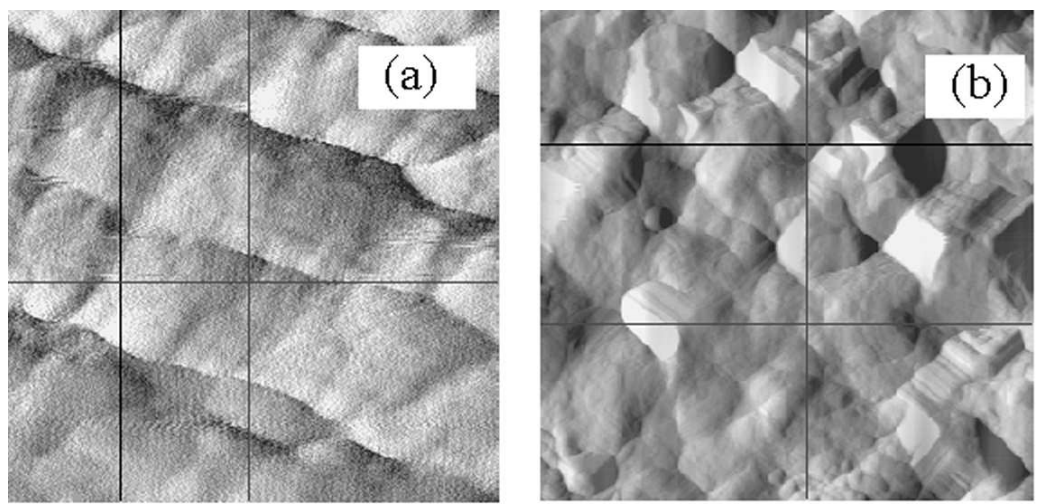

Fig. 3. AFM $1.0 \times 1.0 \mu \mathrm{m}^{2}$ (a) and $10 \times 10 \mu \mathrm{m}^{2}$ (b) micrographs of hybrid samples with $\mathrm{TPPS}_{4}$ deposited on the surface of $c$-Si (a) and por-Si with removed oxide layer (b).

size 10-15 nm [7] were formed during anodic etching. The adsorption of TPPS $_{4}$ on $\mathrm{Si}$ surface is different in all three investigated cases of monocrystalline wafer and por-Si with and without oxide layer. The results obtained demonstrated that por-Si could be perspective media for intercalation of organic molecules like porphyrin.

\section{Acknowledgments}

The work was partly supported by Lithuanian State Science and Studies Foundation (project C-03046).

\section{References}

[1] P. Checcoli, G. Conte, S. Salvatori, R. Paolesse, A. Bolognesi, M. Berliocchi, F. Brunetti, A. D'Amico, A. Di Carlo, P. Lugli, Synth. Met. 138, 261 (2003).

[2] L. Buckberry, S. Bayliss, Materials World 7, 213 (1999).

[3] H. Arwin, M. Gavutis, J. Gustafsson, M. Schultzberg, S. Zangooie, P. Tengvall, Phys. Status Solidi A 182, 515 (2000).

[4] R. Augulis, V. Snitka, R. Rotomskis, Solid State Phenomena 97-98, 191 (2004).

[5] J. Sabataitytè, A. Rèza, I. Šimkienè, A. Matulis, G.-J. Babonas, Solid State Phenomena 97-98, 145 (2004).

[6] I. Šimkiene, Lithuanian J. Phys. 43, 319 (2003).

[7] G. Ambrazevičius, G. Zaicevas, V. Jasutis, D. Leščinskas, T. Lideikis, I. Šimkienė, D. Gulbinaitè, J. Appl. Phys. 76, 5442 (1994). 